\title{
TEACH Cards: Teaching Evidence-Based Medicine and Clinical Topics in the Hospital
}

Thomas W. Hahn, MD | Caitlin D'Agata, MD | Jennifer Edgoose, MD, MPH | Jennifer Mastrocola, MD | Larissa Zakletskaia, MS | Mattie White, MD

PRIMER. 2018;2:25.

Published: 11/14/2018 | DOI: 10.22454/PRiMER.2018.479320

\section{Abstract}

Introduction: Inpatient training and evidence-based medicine (EBM) curricula are fundamental components of medical education. Teaching EBM And Clinical topics in the Hospital (TEACH) Cards is an inpatient curricular tool developed to help guide efficient, discussion-based teaching sessions. TEACH Cards aims to increase frequency of inpatient teaching, improve exposure to the breadth of inpatient topics, advance EBM skills, and improve efficiency in answering clinical questions.

Methods: TEACH Cards is a set of 25 topic-based cards, each addressing an adult inpatient medicine topic by asking background questions and encouraging learners to write and answer foreground questions. Residents and faculty from a family medicine residency rotating on an adult inpatient medicine service during the 6-month study period were invited to complete a prerotation survey, use the TEACH Cards, and then complete a postrotation survey.

Results: Out of 54 potential participants, 35\% completed both the pre- and postrotation surveys. Respondents used TEACH Cards on average three times per week, reporting significantly stronger agreement that they were both learning $(P=0.034)$ and teaching $(P=0.006)$ core inpatient topics. Respondents reported greater confidence in using EBM resources $(P=0.006)$ and significantly shorter time to find an evidence-based answer to a clinical question (pretest median=6-10 minutes vs posttest median=2-5 minutes, $P=0.002$ ).

Conclusion: Use of TEACH Cards increased self-reported exposure to the breadth of core inpatient topics, confidence with EBM skills, and efficiency in finding answers to clinical questions.

\section{Introduction}

Inpatient medicine training is a critical component of family medicine residency education, as approximately $55 \%$ of newly board-certified family physicians plan to provide inpatient care. ${ }^{1,2}$ Inpatient medicine is traditionally taught through didactic sessions and rounds, though the quality of teaching on rounds can vary as it often lacks both learner-centered and evidence-based medicine (EBM) teaching. ${ }^{3}$ Additionally, clinical learners value mini-teaching sessions. $^{4}$

EBM curricula are also important for enhancing ability to answer clinical questions. Traditionally, EBM teaching involves evaluating primary literature in seminars separate from clinical care. ${ }^{5}$ Though isolated EBM teaching improves knowledge, EBM education integrated with clinical care improves skills in evidence appraisal, attitudes of EBM, and EBM behaviors including reading habits, choice of resources, and clinical decision making. ${ }^{6-8}$

We developed Teaching Evidence-based medicine And Clinical topics in the Hospital (TEACH) Cards to enhance inpatient teaching through integrated EBM methods. Each card covers an adult inpatient topic and is a guide for a mini-teaching session facilitated by a faculty or resident physician. Here we present results of a pre/poststudy to 
examine whether using TEACH Cards increases self-reported frequency of inpatient teaching, exposure to adult inpatient topics, ability to write and answer Problem/Patient population, Intervention, Comparison, Outcome (PICO) questions, and efficiency in point-of-care inquiries.

\section{Methods}

Residents and faculty from a university-based family medicine residency participated in this study using pre- and postsurveys. The University Institutional Review Board exempted this study.

\section{Educational Tool}

TEACH Cards are 25 topic-based cards (paper and electronic versions) addressing adult inpatient topics selected from an American Academy of Family Physicians list of topics residents should know to care for critically ill adults. ${ }^{9}$ The front of each card contains background questions about basic science knowledge, a foreground PICO question, and a learning pearl. TEACH Cards also encourage users to write and answer PICO questions. The back of each card provides instructions and resources (Figure 1). Answers to questions are intentionally not provided to encourage self-directed learning and development of EBM skills.

\section{Study Participants and Protocol}

Study participants were family medicine residents and faculty rotating on an adult inpatient service at a community hospital during the 6-month study period. Inpatient rotations were 1-6 weeks in duration; faculty and night residents did 1-2-week rotations and day residents did 4-6 weeks. Potential participants received verbal instructions and an informational email 1 week before the study and an email with electronic surveys the day before and the last day of their rotation. Potential participants could use the TEACH Cards without completing the surveys. Treats were provided for survey completion. Surveys were linked with an anonymous identifier and asked questions regarding inpatient teaching perceptions, EBM skills perceptions, and EBM skills proficiency through writing and answering a PICO question based on a case. Questions 1-5 used a 7-point Likert scale (1=strongly disagree, 7=strongly agree). Participants did not receive a formal orientation to EBM resources, but faculty and senior residents were familiar with the resources from prior experience.

\section{Data Analysis}

We compared pre- and posttest survey continuous responses using one-sample paired $t$-test comparisons of means. Subgroup analysis was performed for resident and faculty respondents. Categorical data were analyzed with a paired-sample Wilcoxon signed rank test.

\section{Results}

Of 54 potential participants, 19 (35\%) completed both pre- and postsurveys: 10 residents (six PGY-1s and four PGY-3s) and nine faculty.

For combined resident and faculty data, six of 10 survey items displayed a statistically significant change in mean response (Table 1). Respondents reported stronger agreement they were learning and teaching core inpatient topics. Respondents acknowledged greater use of EBM resources, greater ability to use EBM resources, decreased time to find an evidence-based answer (median 6-10 minutes vs 2-5 minutes), and greater ability to write a PICO question. On pre- and postsurveys, $100 \%$ of subjects accurately wrote and answered a PICO question.

In resident and faculty subgroup analyses, both groups reported significant increases in ability to write a PICO question and decreased time spent finding an evidence-based answer to a question. Resident respondents reported a significant increase in teaching comprehensive core inpatient topics and a decrease in days per week that topicoriented teaching occurs. Faculty respondents reported significant increases in ability to use evidence-based medicine efficiently to answer clinical questions and in number of days that EBM-guided patient care teaching occurs.

All respondents reported using TEACH Cards, and half used them six or more times. On average, participants used TEACH Cards three times per week, and the majority (67\%) spent 6-15 minutes using one card. Most respondents 
used TEACH Cards for team discussion. Overall, respondents thought TEACH Cards were beneficial for teaching (mean=5.55 on a 1-7 scale [SD 1.19]) and practicing EBM skills (mean=5.55 on a 1-7 scale [SD 1.32]).

\section{Conclusions}

\section{Summary of Findings}

We evaluated an inpatient teaching innovation for family medicine residents and faculty. Pretest ability to accurately write and answer a PICO question was likely due to prior EBM curricula during training. Despite strong baseline EBM skills, there was greater self-reported confidence by residents and faculty in applying EBM skills and using EBM resources after exposure to TEACH Cards. Both residents and faculty reported decreased time to answer EBM questions, which is particularly useful in a busy clinical setting. Furthermore, respondents had greater confidence that they were learning and teaching the breadth of inpatient topics. Subgroup analysis revealed that residents reported teaching more core inpatient topics, whereas faculty reported teaching more EBM-guided patient-care material. It is likely that faculty had more prior experience and confidence teaching core inpatient topics compared to residents, so faculty appreciated TEACH Cards as a tool for teaching EBM skills.

\section{Significance of This Study}

This study supports prior findings that team-based learning is an effective method for mastering EBM skills. ${ }^{10} \mathrm{~A}$ systematic review found that time is the primary barrier for residents practicing EBM, and TEACH Cards may provide a solution, as they appear to promote EBM efficiency. ${ }^{11}$ TEACH Cards can be a useful resource for teaching on a busy inpatient service and may also help with standardization of teaching for inpatient topics. Finally, this instrument provides a means to help meet practice-based learning and improvement milestones. ${ }^{12}$

\section{Study Limitations}

The absence of a control group makes it difficult to determine the full impact of TEACH Cards compared to the inpatient rotation itself. There was a small sample size due to low survey completion rate. Many more people likely utilized TEACH Cards than completed both surveys. There was potential selection bias for people confident in their EBM skills since respondents had high baseline skills.

\section{Conclusion}

TEACH Cards is a promising tool for guiding inpatient teaching and enhancing EBM skills for residents and faculty.

\section{Future Directions}

Future directions include studying TEACH Cards' impact on medical decision-making and expanding TEACH Cards topics. TEACH Cards are available online. ${ }^{13}$

\section{Tables and Figures}




\section{COPD EXACERBATION}

\section{Background Questions:}

$\checkmark$ Define COPD exacerbation.

$\checkmark$ List the key medications for the treatment of mild, moderate, and severe exacerbation.

$\checkmark$ When does a patient need to be admitted to the hospital for treatment of COPD exacerbation?

\section{Foreground Questions:}

$\checkmark$ In hospitalized patients with a COPD exacerbation, do IV or oral corticosteroids lead to decreased mortality?

$\checkmark$ Write your own PICO question, and try to find the answer.

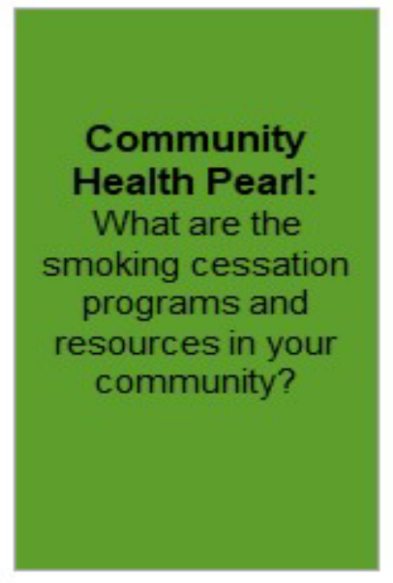

What are the two groups of questions and where can I find answers?

\section{Background Question}

This is a more basic question that answers who, what, where, when, why, and how.

\section{Resources:}

American Family Physician

BMJ Clinical Evidence

DynaMed Plus

Essential Evidence Plus

UpToDate

\section{Foreground Question}

This a specific clinical query: PICO question. Patient population/Problem Intervention/exposure

Comparison (if applicable) Outcome

\section{Resources:}

Cochrane Database

DynaMed Plus

Essential Evidence Plus

PubMed

TRIP Database

What are some uses for the cards?

- To initiate discussion among team members - To guide independent study

- To practice evidence-based medicine

- To create a mini presentation

Answers are intentionally notprovided. 
Table 1: Pretest vs Posttest Scores Comparisons for TEACH Cards Study Surveys ( $N=19)$

\begin{tabular}{|c|c|c|c|}
\hline Questions & $\begin{array}{l}\text { Pretest } \\
\text { Scores } \\
\text { Mean (SD) }\end{array}$ & $\begin{array}{c}\text { Posttest } \\
\text { Scores } \\
\text { Mean (SD) }\end{array}$ & Significance ${ }^{a, b}$ \\
\hline $\begin{array}{l}\text { Q1: I believe I am learning all the core topics required to care for } \\
\text { hospitalized adults. }\end{array}$ & $4.79(1.32)$ & $5.42(0.69)$ & $P=0.048^{*}$ \\
\hline (Residents; N=10) & $4.50(1.58)$ & $5.50(0.53)$ & $P=0.052$ \\
\hline (Faculty; $\mathrm{N}=9$ ) & $5.11(0.93)$ & $5.33(0.87)$ & $P=0.560$ \\
\hline $\begin{array}{l}\text { Q2: I believe I am teaching all the core topics required to care for } \\
\text { hospitalized adults. }\end{array}$ & $4.00(1.49)$ & $4.68(1.29)$ & $P=0.006^{* *}$ \\
\hline (Residents; $\mathrm{N}=10$ ) & $3.20(1.55)$ & $3.90(1.29)$ & $P=0.045^{*}$ \\
\hline (Faculty; N=9) & $4.89(0.78)$ & $5.56(0.53)$ & $P=0.081$ \\
\hline Q3: I can write a PICO question. & $5.47(1.43)$ & $6.53(0.70)$ & $P=0.001^{* *}$ \\
\hline (Residents; N=10) & $5.50(1.65)$ & $6.70(0.67)$ & $P=0.030^{*}$ \\
\hline (Faculty; $N=9$ ) & $5.44(1.24)$ & $6.33(0.71)$ & $P=0.009^{\star *}$ \\
\hline Q4: I use evidence-based resources to answer clinical questions. & $5.63(1.42)$ & $6.26(0.65)$ & $P=0.048^{*}$ \\
\hline (Residents; $\mathrm{N}=10$ ) & $5.60(1.71)$ & $6.40(0.70)$ & $P=0.170$ \\
\hline (Faculty; $\mathrm{N}=9$ ) & $5.67(1.12)$ & $6.11(0.60)$ & $P=0.100$ \\
\hline $\begin{array}{l}\text { Q5: I can use evidence-based medicine resources efficiently to } \\
\text { answer clinical questions. }\end{array}$ & $4.95(1.71)$ & $5.95(0.70)$ & $P=0.006^{* *}$ \\
\hline (Residents; $\mathrm{N}=10$ ) & $4.60(2.17)$ & $5.80(0.79)$ & $P=0.066$ \\
\hline (Faculty; N=9) & $5.33(1.00)$ & $6.11(0.60)$ & $P=0.023^{*}$ \\
\hline $\begin{array}{l}\text { Q6: On average, how many days per week does topic- oriented } \\
\text { teaching occur on the inpatient family medicine service? }\end{array}$ & $4.90(1.73)$ & $4.32(1.42)$ & $P=0.190$ \\
\hline (Residents; $\mathrm{N}=10$ ) & $5.60(1.96)$ & $4.10(1.37)$ & $P=0.043^{*}$ \\
\hline (Faculty; $\mathrm{N}=9$ ) & $4.11(1.05)$ & $4.56(1.51)$ & $P=0.170$ \\
\hline $\begin{array}{l}\text { Q7: On average how many days of the week do you teach topic- } \\
\text { oriented material on the inpatient family medicine service? }\end{array}$ & $3.74(2.31)$ & $3.58(2.01)$ & $P=0.760$ \\
\hline (Residents; N=10) & $3.90(2.96)$ & $3.10(2.13)$ & $P=0.411$ \\
\hline (Faculty; N=9) & $3.56(1.42)$ & $4.11(1.83)$ & $P=0.095$ \\
\hline $\begin{array}{l}\text { Q8: On average how many days per week does EBM-guided patient } \\
\text { care teaching occur on the inpatient family medicine service? }\end{array}$ & $4.58(2.27)$ & $4.53(2.01)$ & $P=0.930$ \\
\hline (Residents; N=10) & $5.80(2.35)$ & $4.70(2.11)$ & $P=0.283$ \\
\hline (Faculty; N=9) & $3.22(1.20)$ & $4.33(1.73)$ & $P=0.030^{*}$ \\
\hline $\begin{array}{l}\text { Q9: On average, how many days of the week do you teach EBM- } \\
\text { guided patient care material on the inpatient service? }\end{array}$ & $3.58(2.52)$ & $3.58(2.01)$ & $P=1.000$ \\
\hline (Residents; N=10) & $4.20(3.19)$ & $3.30(2.41)$ & $P=0.390$ \\
\hline (Faculty; N=9) & $2.89(1.36)$ & $3.89(1.54)$ & $P=0.017^{*}$ \\
\hline $\begin{array}{l}\text { Q10: On average, how many minutes does it take you to find an } \\
\text { evidence-based answer to a clinical question? } \\
<1 \text { minute } \\
2-5 \text { minutes } \\
6-10 \text { minutes } \\
11-15 \text { minutes } \\
>15 \text { minutes }\end{array}$ & $\begin{array}{c}5.3 \% \\
15.8 \% \\
57.9 \% \\
15.8 \% \\
5.3 \%\end{array}$ & $\begin{array}{l}5.3 \% \\
57.9 \% \\
31.6 \% \\
5.3 \% \\
0.0 \% \\
\end{array}$ & $P=0.001^{* *}$ \\
\hline $\begin{array}{l}<1 \text { minute } \\
2-5 \text { minutes } \\
6-10 \text { minutes } \\
11-15 \text { minutes } \\
>15 \text { minutes }\end{array}$ & $\begin{array}{c}10.0 \% \\
0 \% \\
60.0 \% \\
30.0 \% \\
0 \% \\
\end{array}$ & $\begin{array}{l}10.0 \% \\
30.0 \% \\
50.0 \% \\
10.0 \% \\
0.0 \% \\
\end{array}$ & $P=0.015^{\star *}$ \\
\hline $\begin{array}{l}<1 \text { minute } \\
2-5 \text { minutes } \\
6-10 \text { minutes } \\
11-15 \text { minutes } \\
>15 \text { minutes }\end{array}$ & $\begin{array}{c}0 \% \\
33.3 \% \\
55.6 \% \\
0 \% \\
11.1 \%\end{array}$ & $\begin{array}{c}0 \% \\
88.9 \% \\
11.1 \% \\
0 \% \\
0 \%\end{array}$ & $P=0.008^{* *}$ \\
\hline
\end{tabular}

${ }^{*} P<0.05$. 
${ }^{* *} P<0.01$.

a One sample t-test.

${ }^{\mathrm{b}} \mathrm{A}$ paired-sample Wilcoxon signed rank test.

\section{Acknowledgments}

The authors thank Jay Brieler, MD, and Sarah Hilding, MD, for assistance with research; John Frey, MD, and Craig Gjerde, PhD, for guidance with conceptual development; Amy E. LaVertu, MLS, for assistance with the literature review; Mindy Smith, MD, MS, for guidance with the manuscript; and James Witkins, BA, for creating the TEACH Cards website.

Financial Support: Financial support was provided by the University of Wisconsin Department of Family Medicine and Community Health through the academic fellowship scholarly project fund.

\section{Presentations:}

Hahn T, Edgoose J, D'Agata C, Hilding S, Brieler J, White M. TEACH Cards: a multi-center look at inpatient EBM teaching and PBLI-1 milestone mastery. 2015 Society of Teachers of Family Medicine Annual Spring Conference. April 25-29, Orlando, FL.

Edgoose J, D'Agata C, Hahn T, Mastrocola J. Inpatient TEACH Cards: striving toward practice-based learning and improvement (PBLI). 2014 Society of Teachers of Family Medicine Annual Spring Conference. May 3-7, 2014. San Antonio, TX.

\section{Corresponding Author}

Thomas W. Hahn, MD

100 N Nine Mound Rd, Verona, WI 53593. 608-395-1666. Fax: 608-845-8684. thomas.hahn@fammed.wisc.edu.

\section{Author Affiliations}

Thomas W. Hahn, MD - Department of Family Medicine and Community Health, University of Wisconsin School of Medicine and Public Health

Caitlin D'Agata, MD - Department of Family Medicine, Tufts University School of Medicine

Jennifer Edgoose, MD, MPH - Department of Family Medicine and Community Health, University of Wisconsin

School of Medicine and Public Health

Jennifer Mastrocola, MD - Department of Family Medicine, University of Connecticut School of Medicine

Larissa Zakletskaia, MS - Department of Family Medicine and Community Health, University of Wisconsin School of Medicine and Public Health

Mattie White, MD - Department of Family and Community Medicine, Saint Louis University School of Medicine

\section{References}

1. Wiest FC, Ferris TG, Gokhale M, Campbell EG, Weissman JS, Blumenthal D. Preparedness of internal medicine and family practice residents for treating common conditions. JAMA. 2002;288(20):2609-2614. https://doi.org/10.1001/jama.288.20.2609

2. Coutinho AJ, Cochrane A, Stelter K, Phillips RL Jr, Peterson LE. Comparison of intended scope of practice for family medicine residents with reported scope of practice among practicing family physicians. JAMA. 2015;314(22):2364-2372. https://doi.org/10.1001/jama.2015.13734

3. Stickrath C, Noble M, Prochazka A, et al. Attending rounds in the current era: what is and is not happening. JAMA Intern Med. 2013;173(12):1084-1089. https://doi.org/10.1001/jamainternmed.2013.6041

4. Torre DM, Simpson D, Sebastian JL, Elnicki DM. Learning/feedback activities and high-quality teaching: perceptions of third-year medical students during an inpatient rotation. Acad Med. 2005;80(10):950-954. https://doi.org/10.1097/00001888-200510000-00016

5. Green ML. Graduate medical education training in clinical epidemiology, critical appraisal, and evidence-based medicine: a critical review of curricula. Acad Med. 1999;74(6):686-694. https://doi.org/10.1097 
6. Coomarasamy A, Khan KS. What is the evidence that postgraduate teaching in evidence based medicine changes anything? A systematic review. BMJ. 2004;329(7473):1017-1021. https://doi.org/10.1136 /bmj.329.7473.1017

7. Allan GM, Korownyk C, Tan A, Hindle H, Kung L, Manca D. Developing an integrated evidence-based medicine curriculum for family medicine residency at the University of Alberta. Acad Med. 2008;83(6):581-587. https://doi.org/10.1097/ACM.0b013e3181723a5c

8. Shaughnessy AF, Gupta PS, Erlich DR, Slawson DC. Ability of an information mastery curriculum to improve residents' skills and attitudes. Fam Med. 2012;44(4):259-264.

9. American Academy of Family Physicians. Reprint No AAFP 291. Recommended curriculum guidelines for family practice residents: Care of the critically ill adult. Published June, 2003. Revised June 2015.

10. Hunt DP, Haidet $P$, Coverdale JH, Richards $B$. The effect of using team learning in an evidence-based medicine course for medical students. Teach Learn Med. 2003;15(2):131-139. https://doi.org/10.1207 /S15328015TLM1502_11

11. van Dijk N, Hooft L, Wieringa-de Waard $M$. What are the barriers to residents' practicing evidence-based medicine? A systematic review. Acad Med. 2010;85(7):1163-1170. https://doi.org/10.1097 /ACM.0b013e3181d4152f

12. The Family Medicine Milestone Project. Journal of Graduate Medical Education: March 2014, Vol. 6, No. 1s1, pp. 74-86. https://doi.org/10.4300/JGME-06-01s1-05

13. University of Wisconsin Department of Family Medicine and Community Health. TEACH Cards. https://www.fammed.wisc.edu/teachcards. Accessed July 1, 2018.

Copyright $(92018$ by the Society of Teachers of Family Medicine 